\title{
Nonrenal Clearance Normalized by BMI
}

National Cancer Institute

\section{Source}

National Cancer Institute. Nonrenal Clearance Normalized by BMI. NCI Thesaurus. Code C105454.

The total clearance of a substance from the blood minus the renal clearance divided by the body mass index. 